\title{
Bottom-up Thermal Plasma Approach for Graphene: Tuning catalyst materials for PEM-FC
}

\author{
J-L. Meunier \\ Department of Chemical Engineering \\ Plasma Processing Laboratory (PPL) \\ McGill University \\ Montreal, Canada \\ Jean-Luc.Meunier@mcgill.ca
}

Thermal plasma (TP) reactors are used for the generation of particles having specific compositions or phase structures, while nanoparticles (NPs) are also being generated using precursors that are either in the gas phase, in liquid solutions or even sometimes in the solid phase. More difficult is the controlled homogeneous nucleation of pure nanomaterials with specific structures, or the controlled two-step systems for heterogeneous nucleation of materials such as carbon nanotubes (CNTs).

One material of strong interest is the bi-dimensional structure of graphene. This talk presents first the bottom-up approach for generating graphene structures from the controlled homogeneous nucleation and rapid 2D growth of pure graphene powders from the gas phase. The specific application in mind relates to PEM-Fuel Cell catalysts, setting specific requirements on both the structure and the functionalities to be imbedded on the graphene material. The thermal plasma processing route generates exceptionally high levels of crystallinity, purity and "tunability" for in situ chemical functionalization in specific applications. To achieve this, a strong control of the flow, energy, chemistry and particles nucleation fields is essential in the reactor, the nucleation itself occurring within an extreme temperature window in the plasma between $4500 \mathrm{~K}$ and $5000 \mathrm{~K}$. A first part of the talk will relate to achieving an understanding of this controlled environment, and the second part to the catalyst generated tested in a PEM-FC environment. 